\title{
AN APPROACH TO PRE-SEPARATION AXIOMS IN NEUTROSOPHIC SOFT TOPOLOGICAL SPACES
}

\author{
Ahu AÇIKGÖZ and Ferhat ESENBEL \\ Department of Mathematics, Balikesir University, 10145 Balikesir, TURKEY
}

\begin{abstract}
In this study, we introduce the concept of neutrosophic soft preopen (neutrosophic soft pre-closed) sets and pre-separation axioms in neutrosophic soft topological spaces. In particular, the relationship between these separation axioms are investigated. Also, we give a new definition for neutrosophic soft topological subspace and define neutrosophic soft pre irresolute soft and neutrosophic pre irresolute open soft functions.
\end{abstract}

\section{INTRODUCTION}

In 2005, Smarandache introduced the concept of a neutrosophic set 20 as a generalization of classical sets, fuzzy set theory 20] (see also [10]), intuitionistic fuzzy set theory [4] (see also 14]) etc. By using this theory of neutrosophic set, some scientists made researches in many areas of mathematics [7,18. Many inherent difficulties exist in classical methods for the inadequacy of the theories of parametrization tools. So, classical methods are insufficient in dealing with several practical problems in some other disciplines such as economics, engineering, environment, social science, medical science, etc. In 1999, Molodtsov 16 pointed out the inherent difficulties of these theories. A different approach was initiated by Molodtsov for modeling uncertainties. This approach was applied in some other directions such as smoothness of functions, game theory, operations research, Riemann integration, Perron integration and so on. The theory of soft topological spaces was introduced by Shabir and Naz [19] for the first time in 2011. Soft topological spaces were defined over an initial universe with a fixed set of parameters and showed that a soft topological space gives a parameterized family of topological

2020 Mathematics Subject Classification. 54A05, 54D10, 54D15.

Keywords and phrases. Neutrosophic pre open soft set, neutrosophic soft pre interior point, neutrosophic soft pre cluster point, neutrosophic soft pre-separation axioms, neutrosophic soft subspace.

\ahuacikgoz@gmail.com-Corresponding author; fesenbel@gmail.com

(D) 0000-0003-1468-8240; 0000-0001-2345-6789.

(C)2020 Ankara University Communications Faculty of Sciences University of Ankara-Series A1 Mathematics and Statistics 
spaces. In 1, 2, 5, 6, 9, 11, 13], some scientists made researches and did theoretical studies in soft topological spaces. In 2013, Maji [15] defined the concept of neutrosophic soft sets for the first time. Then, Deli and Broumi [12] modified this concept. In 2017, Bera presented neutrosophic soft topological spaces in [8].

In this study, our pupose is to adapt the concepts of neutrosophic pre open soft set, neutrosophic pre closed soft set to neutrosophic soft topological spaces. Then, we define neutrosophic soft pre interior point, neutrosophic soft pre cluster point, neutrosophic soft pre interior operator and neutrosophic soft pre closure operator. By using these definitions and concepts, the concept of pre-separation axioms of neutrosophic soft topological spaces is introduced. Furthermore, we analyze properties of neutrosophic soft pre $\mathrm{T}_{i}$-spaces $(i=0,1,2,3,4)$ and focus on some relations between them. Characterization theorems of them are also proved. We hope that, the findings in this document will help scientists to enhance and promote the further studies on neutrosophic soft topology to carry out a general framework for their applications in practical life.

\section{Preliminaries}

In this section, we present the basic definitions and theorems related to neutrosophic soft set theory.

Definition 1. [20] $A$ neutrosophic set $A$ on the universe set $X$ is defined as:

$A=\left\{\left\langle x, T_{A}(x), I_{A}(x), F_{A} x\right\rangle: x \in X\right\}$,

where

$T, I, F: X \rightarrow]^{-} 0,1^{+}\left[\right.$and $^{-} 0 \leq T_{A}(x)+I_{A}(x)+F_{A}(x) \leq 3^{+}$.

Definition 2. [16] Let $X$ be an initial universe, $E$ be a set of all parameters, and $P(X)$ denote the power set of $X . A$ pair $(F, E)$ is called a soft set over $X$, where $F$ is a mapping given by $F: E \rightarrow P(X)$. In other words, the soft set is a parameterized family of subsets of the set $X$. For $e \in E, F(e)$ may be considered as the set of e-elements of the soft set $(F, E)$, or as the set of e-approximate elements of the soft set, i.e.

$(F, E)=\{(e, F(e)): e \in E, F: E \rightarrow P(X)\}$.

After the neutrosophic soft set was defined by Maji [15], this concept was modified by Deli and Broumi [12] as given below:

Definition 3. [12] Let $X$ be an initial universe set and $E$ be a set of parameters. Let $P(X)$ denote the set of all neutrosophic sets of $X$. Then a neutrosophic soft set $(\widetilde{F}, E)$ over $X$ is a set defined by a set valued function $\widetilde{F}$ representing a mapping $\widetilde{F}: E \rightarrow P(X)$, where $\widetilde{F}$ is called the approximate function of the neutrosophic soft

set $(\widetilde{F}, E)$. In other words, the neutrosophic soft set is a parametrized family of some elements of the set $P(X)$ and therefore it can be written as a set of ordered pairs: 


$$
(\widetilde{F}, E)=\left\{\left(e,\left\langle x, T_{\widetilde{F}(e)}(x), I_{\widetilde{F}(e)}(x), F_{\widetilde{F}(e)}(x)\right\rangle: x \in X\right): e \in E\right\}
$$

where $T_{\widetilde{F}(e)}(x), I_{\widetilde{F}(e)}(x), F_{\widetilde{F}(e)}(x) \in[0,1]$ are respectively called the truthmembership,

indeterminacy-membership and falsity-membership function of $\widetilde{F}(e)$. Since the supremum of each $T, I, F$ is 1 , the inequality

$0 \leq T_{A}(x)+I_{A}(x)+F_{A}(x) \leq 3$

is obvious.

Definition 4. [8] Let $(\widetilde{F}, E)$ be a neutrosophic soft set over the universe set $X$. The complement of $(\widetilde{F}, E)$ is denoted by $(\widetilde{F}, E)^{c}$ and is defined by:

$$
(\widetilde{F}, E)^{c}=\left\{\left(e,\left\langle x, F_{\widetilde{F}(e)}(x), 1-I_{\widetilde{F}(e)}(x), T_{\widetilde{F}(e)}(x)\right\rangle: x \in X\right): e \in E\right\} .
$$

It is obvious that $\left[(\widetilde{F}, E)^{c}\right]^{c}=(\widetilde{F}, E)$.

Definition 5. [15] Let $(\widetilde{F}, E)$ and $(\widetilde{G}, E)$ be two neutrosophic soft sets over the universe set $X .(\widetilde{F}, E)$ is said to be a neutrosophic soft subset of $(\widetilde{G}, E)$ if

$T_{\widetilde{F}(e)}(x) \leq T_{\widetilde{G}(e)}(x), I_{\widetilde{F}(e)}(x) \leq I(x), F_{\widetilde{F}(e)}(x) \geq F_{\widetilde{G}(e)}(x), \forall e \in E, \forall x \in X$.

It is denoted by $(\widetilde{F}, E) \subseteq(\widetilde{G}, E)$. ( $\widetilde{F}, E)$ is said to be neutrosophic soft equal to $(\widetilde{G}, E)$ if $(\widetilde{F}, E) \subseteq(\widetilde{G}, E)$ and $(\widetilde{G}, E) \subseteq(\widetilde{F}, E)$. It is denoted by $(\widetilde{F}, E)=$ $(\widetilde{G}, E)$.

Definition 6. [3] Let $\left(\widetilde{F}_{1}, E\right)$ and $\left(\widetilde{F}_{2}, E\right)$ be two neutrosophic soft sets over the universe set $X$. Then their union is denoted by $\left(\widetilde{F}_{1}, E\right) \cup\left(\widetilde{F}_{2}, E\right)=\left(\widetilde{F}_{3}, E\right)$ and is defined by:

$$
\begin{aligned}
& \left(\widetilde{F}_{3}, E\right)=\left\{\left(e,\left\langle x, T_{\widetilde{F}_{3}(e)}(x), I(x), F_{\widetilde{F}_{3}(e)}(x)\right\rangle: x \in X\right): e \in E\right\}, \\
& \text { where } \\
& T_{\widetilde{F}_{3}(e)}(x)=\max \left\{T_{\widetilde{F}_{1}(e)}(x), T_{\widetilde{F}_{2}(e)}(x)\right\}, \\
& I_{\widetilde{F}_{3}(e)}(x)=\max \left\{I_{\widetilde{F}_{1}(e)}(x), I_{\widetilde{F}_{2}(e)}(x)\right\}, \\
& F_{\widetilde{F}_{3}(e)}(x)=\min \left\{F_{\widetilde{F}_{1}(e)}(x), F_{\widetilde{F}_{2}(e)}(x)\right\} .
\end{aligned}
$$

Definition 7. 3. Let $\left(\widetilde{F}_{1}, E\right)$ and $\left(\widetilde{F}_{2}, E\right)$ be two neutrosophic soft sets over the universe set $X$. Then their intersection is denoted by $\left(\widetilde{F}_{1}, E\right) \cap\left(\widetilde{F}_{2}, E\right)=\left(\widetilde{F}_{3}, E\right)$ and is defined by:

$$
\begin{aligned}
& \left(\widetilde{F}_{3}, E\right) \\
& \text { where }
\end{aligned}=\left\{\left(e,\left\langle x, T_{\widetilde{F}_{3}(e)}(x), I(x), F_{\widetilde{F}_{3}(e)}(x)\right\rangle: x \in X\right): e \in E\right\},
$$




$$
\begin{aligned}
& T_{\widetilde{F}_{3}(e)}(x)=\min \left\{T_{\widetilde{F}_{1}(e)}(x), T_{\widetilde{F}_{2}(e)}(x)\right\}, \\
& I_{\widetilde{F}_{3}(e)}(x)=\min \left\{I_{\widetilde{F}_{1}(e)}(x), I_{\widetilde{F}_{2}(e)}(x)\right\}, \\
& F_{\widetilde{F}_{3}(e)}(x)=\max \left\{F_{\widetilde{F}_{1}(e)}(x), F_{\widetilde{F}_{2}(e)}(x)\right\} .
\end{aligned}
$$

Definition 8. 3. A neutrosophic soft set $(\widetilde{F}, E)$ over the universe set $X$ is said to be a null neutrosophic soft set if $T_{\widetilde{F}(e)}(x)=0, I_{\widetilde{F}(e)}(x)=0, F_{\widetilde{F}(e)}(x)=1$; $\forall e \in E, \forall x \in X$. It is denoted by $0_{(X, E)}$.

Definition 9. 3. A neutrosophic soft set $(\widetilde{F}, E)$ over the universe set $X$ is said to be an absolute neutrosophic soft set if $T_{\widetilde{F}(e)}(x)=1, I_{\widetilde{F}(e)}(x)=1, F_{\widetilde{F}(e)}(x)=0$; $\forall e \in E, \forall x \in X$ It is denoted by $1_{(X, E)}$.

Clearly $0_{(X, E)}^{c}=1_{(X, E)}$ and $1_{(X, E)}^{c}=0_{(X, E)}$.

Definition 10. [3] Let NSS $(X, E)$ be the family of all neutrosophic soft sets over the universe set $\bar{X}$ and $\tau \subset N S S(X, E)$. Then $\tau$ is said to be a neutrosophic soft topology on $X$ if:

1. $0_{(X, E)}$ and $1_{(X, E)}$ belong to $\tau$,

2. the union of any number of neutrosophic soft sets in $\tau$ belongs to $\tau$,

3. the intersection of a finite number of neutrosophic soft sets in $\tau$ belongs to $\tau$.

Then $(X, \tau, E)$ is said to be a neutrosophic soft topological space over $X$. Each member of $\tau$ is said tobe a neutrosophic soft open set [3].

Definition 11. 3et $(X, \tau, E)$ be a neutrosophic soft topological space over $X$ and $(\widetilde{F}, E)$ be a neutrosophic soft set over $X$. Then $(\widetilde{F}, E)$ is said to be a neutrosophic soft closed set iff its complement is a neutrosophic soft open set.

Definition 12. [3] Let NSS $(X, E)$ be the family of all neutrosophic soft sets over the universe set $X$. Then neutrosophic soft set $x_{(\alpha, \beta, \gamma)}^{e}$ is called a neutrosophic soft point for every $x \in X, 0<\alpha, \beta, \gamma \leq 1, e \in E$ and is defined as follows:

$$
x_{(\alpha, \beta, \gamma)}^{e}\left(e^{\prime}\right)(y)= \begin{cases}(\alpha, \beta, \gamma), & \text { if } e^{\prime}=e \text { and } y=x \\ (0,0,1), & \text { if } e^{\prime} \neq e \text { or } y \neq x\end{cases}
$$

It is clear that every neutrosophic soft set is the union of its neutrosophic soft points.

Definition 13. 3. Let $(\widetilde{F}, E)$ be a neutrosophic soft set over the universe set $X$. We say that $x_{(\alpha, \beta, \gamma)}^{e} \in(\widetilde{F}, E)$ read as belonging to the neutrosophic soft $\operatorname{set}(\widetilde{F}, E)$ whenever

$\alpha \leq T_{\widetilde{F}(e)}(x), \beta \leq I_{\widetilde{F}(e)}(x)$ and $\gamma \geq F_{\widetilde{F}(e)}(x)$. 
Definition 14. 3et $x_{(\alpha, \beta, \gamma)}^{e}$ and $y_{\left(\alpha^{\prime}, \beta^{\prime}, \gamma^{\prime}\right)}^{e^{\prime}}$ be two neutrosophic soft points. For the neutrosophic soft points $x_{(\alpha, \beta, \gamma)}^{e}$ and $y_{\left(\alpha^{\prime}, \beta^{\prime}, \gamma^{\prime}\right)}^{e^{\prime}}$ over a common universe $X$, we say that the neutrosophic soft points are distinct points if $x_{(\alpha, \beta, \gamma)}^{e} \cap y_{\left(\alpha^{\prime}, \beta^{\prime}, \gamma^{\prime}\right)}^{e^{\prime}}=$ $0_{(X, E)}$. It is clear that $x_{(\alpha, \beta, \gamma)}^{e}$ and $y_{\left(\alpha^{\prime}, \beta^{\prime}, \gamma^{\prime}\right)}^{e^{\prime}}$ are distinct neutrosophic soft points if and only if $x \neq y$ or $e \neq e^{\prime}$.

Definition 15. Let $\left(\widetilde{F}, E_{1}\right),\left(\widetilde{G}, E_{2}\right)$ be two neutrosophic sets over the universal set $X$. Then their cartesian product is another neutrosophic set $\left(\widetilde{K}, E_{3}\right)=$ $\left(\widetilde{F}, E_{1}\right) \times\left(\widetilde{G}, E_{2}\right)$, where $E_{3}=E_{1} \times E_{2}$ and $\widetilde{K}\left(e_{1}, e_{2}\right)=\widetilde{F}\left(e_{1}\right) \times \widetilde{G}\left(e_{2}\right)$. The truth, indeterminacy and falsity membership of $\left(\widetilde{K}, E_{3}\right)$ are given by $\forall e_{1} \in E_{1}$, $\forall e_{2} \in E_{2}, \forall x \in X$,

$$
\begin{aligned}
& T_{\widetilde{K}\left(e_{1}, e_{2}\right)}(x)=\min \left\{T_{\widetilde{F}\left(e_{1}\right)}(x), T_{\widetilde{G}\left(e_{2}\right)}(x)\right\}, \\
& I_{\widetilde{K}\left(e_{1}, e_{2}\right)}(x)=\min \left\{I_{\widetilde{F}\left(e_{1}\right)}(x), I_{\widetilde{G}\left(e_{2}\right)}(x)\right\}, \\
& F_{\widetilde{K}\left(e_{1}, e_{2}\right)}(x)=\max \left\{F_{\widetilde{F}\left(e_{1}\right)}(x), F_{\widetilde{G}\left(e_{2}\right)}(x)\right\} .
\end{aligned}
$$

This definition can be extended for more than two neutrosophic soft sets.

Definition 16. [7] A neutrosophic soft relation $\widetilde{R}$ between two neutrosophic soft sets $\left(\widetilde{F}, E_{1}\right)$ and $\left(\widetilde{G}, E_{2}\right)$ over the common universe $X$ is the neutrosophic soft subset of $\left(\widetilde{F}, E_{1}\right) \times\left(\widetilde{G}, E_{2}\right)$. Clearly, it is another neutrosophic soft set $\left(\widetilde{R}, E_{3}\right)$ where $E_{3} \subset E_{1} \times E_{2}$ and

$$
\widetilde{R}\left(e_{1}, e_{2}\right)=\widetilde{F}\left(e_{1}\right) \times \widetilde{G}\left(e_{2}\right) \text { for }\left(e_{1}, e_{2}\right) \in E_{3} .
$$

Definition 17. [7] Let $\left(\widetilde{F}, E_{1}\right),\left(\widetilde{G}, E_{2}\right)$ be two neutrosophic sets over the universal set $X$ and $f$ be a neutrosophic soft relation defined on $\left(\widetilde{F}, E_{1}\right) \times\left(\widetilde{G}, E_{2}\right)$. Then $f$ is called neutrosophic soft function if $f$ associates each element of $\left(\widetilde{F}, E_{1}\right)$ with the unique element of $\left(\widetilde{G}, E_{2}\right)$. We write $f:\left(\widetilde{F}, E_{1}\right) \rightarrow\left(\widetilde{G}, E_{2}\right)$ as a neutrosophic soft function or a mapping. For $x_{(\alpha, \beta, \gamma)}^{e} \in\left(\widetilde{F}, E_{1}\right)$ and $y_{\left(\alpha^{\prime}, \beta^{\prime}, \gamma^{\prime}\right)}^{e^{\prime}} \in\left(\widetilde{G}, E_{2}\right)$ when $x_{(\alpha, \beta, \gamma)}^{e} \times y_{\left(\alpha^{\prime}, \beta^{\prime}, \gamma^{\prime}\right)}^{e^{\prime}} \in f$, we denote it by $f\left(x_{(\alpha, \beta, \gamma)}^{e}\right)=y_{\left(\alpha^{\prime}, \beta^{\prime}, \gamma^{\prime}\right)}^{e^{\prime}}$. Here $\left(\widetilde{F}, E_{1}\right)$ and $\left(\widetilde{G}, E_{2}\right)$ are called domain and codomain respectively and $y_{\left(\alpha^{\prime}, \beta^{\prime}, \gamma^{\prime}\right)}^{e^{\prime}}$ is the image of $x_{(\alpha, \beta, \gamma)}^{e}$ under $f$. 
Definition 18. [8] Let $(X, \tau, E)$ be a neutrosophic soft topological space and $(\widetilde{F}, E) \in N S S(X, E)$ be arbitrary. Then the interior of $(\widetilde{F}, E)$ is denoted by $(\widetilde{F}, E)^{\circ}$ and is defined as:

$(\widetilde{F}, E)^{\circ}=\bigcup\{(\widetilde{G}, E):(\widetilde{G}, E) \subset(\widetilde{F}, E), \quad(\widetilde{G}, E) \in \tau\}$

i.e., it is the union of all open neutrosophic soft subsets of $(\widetilde{F}, E)$.

Definition 19. $[8]$ Let $(X, \tau, E)$ be a neutrosophic soft topological space and $(\widetilde{F}, E) \in N S S(X, E)$ be arbitrary. Then the closure of $(\widetilde{F}, E)$ is denoted by $\overline{(\widetilde{F}, E)}$ and is defined as:

$\overline{(\widetilde{F}, E)}=\bigcap\left\{(\widetilde{G}, E):(\widetilde{G}, E) \subset(\widetilde{F}, E),(\widetilde{G}, E)^{c} \in \tau\right\}$ i.e., it is the intersection of all closed neutrosophic soft super sets of $(\widetilde{F}, E)$.

\section{Some Properties}

Definition 20. A subset $(\widetilde{F}, E)$ of a neutrosophic soft topological space $(X, \tau, E)$ is said to be neutrosophic pre open soft, if $(\widetilde{F}, E) \subset[\overline{(\widetilde{F}, E)}]^{\circ}$. The family of all neutrosophic pre open soft sets of $(X, \tau, E)$ is denoted by $N \operatorname{SPO}(X)$. The family of all neutrosophic pre open soft sets of $(X, \tau, E)$ containing a neutrosophic soft point $x_{(\alpha, \beta, \gamma)}^{e}$ is denoted by NSPO $\left(X, x_{(\alpha, \beta, \gamma)}^{e}\right)$.

Definition 21. A neutrosophic soft point $x_{(\alpha, \beta, \gamma)}^{e}$ of a neutrosophic soft topological space

$(X, \tau, E)$ is said to be neutrosophic soft pre interior point of a neutrosophic soft set $(\widetilde{F}, E)$, if there exists $(\widetilde{G}, E) \in N \operatorname{SPO}\left(X, x_{(\alpha, \beta, \gamma)}^{e}\right)$ such that $x_{(\alpha, \beta, \gamma)}^{e} \nsubseteq(\widetilde{G}, E)^{c}$ and $(\widetilde{G}, E) \subset(\widetilde{F}, E)$.

Definition 22. The set of all neutrosophic soft pre interior points of $(\widetilde{F}, E)$ is said to be neutrosophic soft pre interior of $(\widetilde{F}, E)$ and denoted by $N \operatorname{SPint}(\widetilde{F}, E)$.

Definition 23. The complement of a neutrosophic pre open soft set is called neutrosophic pre closed soft. The intersection of all neutrosophic pre closed soft sets containing a neutrosophic soft set $(\widetilde{F}, E)$ is called neutrosophic pre closure of $(\widetilde{F}, E)$ and is denoted by $\operatorname{NSPcl}(\widetilde{F}, E)$.

Definition 24. A neutrosophic soft point $x_{(\alpha, \beta, \gamma)}^{e}$ of a neutrosophic soft topological space 
$(X, \tau, E)$ is said to be neutrosophic soft pre cluster point of a neutrosophic soft set $(\widetilde{F}, E)$, if $(\widetilde{G}, E) \nsubseteq(\widetilde{F}, E)^{c}$ for any $(\widetilde{G}, E) \in N S P O\left(X, x_{(\alpha, \beta, \gamma)}^{e}\right)$.

Definition 25. A neutrosophic soft topological space $(X, \tau, E)$ is said to be a neutrosophic soft pre $T_{0}$-space if for every pair of distinct neutrosophic soft points $x_{(\alpha, \beta, \gamma)}^{e}, y_{\left(\alpha^{\prime}, \beta^{\prime}, \gamma^{\prime}\right)}^{e^{\prime}}$ there exist neutrosophic pre-open soft sets $(\widetilde{F}, E),(\widetilde{G}, E)$ such that $x_{(\alpha, \beta, \gamma)}^{e} \in(\widetilde{F}, E)$,

$y_{\left(\alpha^{\prime}, \beta^{\prime}, \gamma^{\prime}\right)}^{e^{\prime}} \in(\widetilde{F}, E)^{c}$ or $x_{(\alpha, \beta, \gamma)}^{e} \in(\widetilde{G}, E)^{c}, y_{\left(\alpha^{\prime}, \beta^{\prime}, \gamma^{\prime}\right)}^{e^{\prime}} \in(\widetilde{G}, E)$.

Definition 26. Let $(X, \tau, E)$ be a neutrosophic soft topological space and $Y \subseteq X$. Let $(\widetilde{H}, E)$ be a neutrosophic soft set over $Y$ such that

$$
\begin{aligned}
& T_{\widetilde{H}(e)}(x)= \begin{cases}1, & \text { if } x \in Y \\
0, & \text { if } x \notin Y\end{cases} \\
& I_{\widetilde{H}(e)}(x)= \begin{cases}1, & \text { if } x \in Y \\
0, & \text { if } x \notin Y\end{cases} \\
& F_{\widetilde{H}(e)}(x)= \begin{cases}1, & \text { if } x \in Y \\
0, & \text { if } x \notin Y\end{cases}
\end{aligned}
$$

for any $e \in E$.

Let $\tau_{Y}=\{(\widetilde{H}, E) \cap(\widetilde{F}, E):(\widetilde{F}, E) \in \tau\}$, then $\left(Y, \tau_{Y}, E\right)$ is called neutrosophic soft subspace of $(X, \tau, E)$. If $(\widetilde{H}, E) \in \tau$ (resp. $\left.(\widetilde{H}, E)^{c} \in \tau\right)$, then $\left(Y, \tau_{Y}, E\right)$ is called neutrosophic open (resp.closed) soft subspace of $(X, \tau, E)$.

Theorem 27. A neutrosophic soft subspace $\left(Y, \tau_{Y}, E\right)$ of a neutrosophic soft pre $T_{0}$-space $(X, \tau, E)$ is neutrosophic soft pre $T_{0}$.

Proof. Let $x_{(\alpha, \beta, \gamma)}^{e}, y_{\left(\alpha^{\prime}, \beta^{\prime}, \gamma^{\prime}\right)}^{e^{\prime}}$ be two distinct neutrosophic soft points in $\left(Y, \tau_{Y}, E\right)$. Then, these neutrosophic soft points are also in $(X, \tau, E)$. Hence, there exist neutrosophic pre-open soft sets $(\widetilde{F}, E),(\widetilde{G}, E)$ in $\tau$ such that $x_{(\alpha, \beta, \gamma)}^{e} \in(\widetilde{F}, E)$, $y_{\left(\alpha^{\prime}, \beta^{\prime}, \gamma^{\prime}\right)}^{e^{\prime}} \in(\widetilde{F}, E)^{c}$ or $x_{(\alpha, \beta, \gamma)}^{e} \in(\widetilde{G}, E)^{c}, y_{\left(\alpha^{\prime}, \beta^{\prime}, \gamma^{\prime}\right)}^{e^{\prime}} \in(\widetilde{G}, E)$. Let $(\widetilde{H}, E)$ be a neutrosophic soft set over $Y$ as described in Definition 26. Thus, $(\widetilde{H}, E) \cap(\widetilde{F}, E)$ and $(\widetilde{H}, E) \cap(\widetilde{G}, E)$ are neutrosophic pre-open soft sets in $\left(Y, \tau_{Y}, E\right)$ such that $x_{(\alpha, \beta, \gamma)}^{e} \in(\widetilde{H}, E) \cap(\widetilde{F}, E), y_{\left(\alpha^{\prime}, \beta^{\prime}, \gamma^{\prime}\right)}^{e^{\prime}} \in[(\widetilde{H}, E) \cap(\widetilde{F}, E)]^{c}$ or 
$x_{(\alpha, \beta, \gamma)}^{e} \in[(\widetilde{H}, E) \cap(\widetilde{G}, E)]^{c}, y_{\left(\alpha^{\prime}, \beta^{\prime}, \gamma^{\prime}\right)}^{e^{\prime}} \in(\widetilde{H}, E) \cap(\widetilde{G}, E)$. Therefore, $\left(Y, \tau_{Y}, E\right)$ is neutrosophic soft pre $T_{0}$.

Definition 28. A neutrosophic soft topological space $(X, \tau, E)$ is said to be a neutrosophic soft pre $T_{1}$-space if for every pair of distinct neutrosophic soft points $x_{(\alpha, \beta, \gamma)}^{e}, y_{\left(\alpha^{\prime}, \beta^{\prime}, \gamma^{\prime}\right)}^{e^{\prime}}$ there exists neutrosophic pre-open soft sets $(\widetilde{F}, E)$ and $(\widetilde{G}, E)$ such that $x_{(\alpha, \beta, \gamma)}^{e} \in(\widetilde{F}, E)$, $y_{\left(\alpha^{\prime}, \beta^{\prime}, \gamma^{\prime}\right)}^{e^{\prime}} \in(\widetilde{F}, E)^{c}$ and $x_{(\alpha, \beta, \gamma)}^{e} \in(\widetilde{G}, E)^{c}, y_{\left(\alpha^{\prime}, \beta^{\prime}, \gamma^{\prime}\right)}^{e^{\prime}} \in(\widetilde{G}, E)$.

Theorem 29. A neutrosophic soft subspace $\left(Y, \tau_{Y}, E\right)$ of a neutrosophic soft pre $T_{1}$-space $(X, \tau, E)$ is neutrosophic soft pre $T_{1}$

Proof. It is similar to the proof of Theorem 27.

Theorem 30. Every neutrosophic soft point with the truth-membership value 1, the indeterminacy-membership value 1 and falsity-membership value 0 , is neutrosophic pre-closed soft in a neutrosophic soft topological space $(X, \tau, E)$ if and only if $(X, \tau, E)$ is neutrosophic soft pre $T_{1}$.

Proof. $(\Rightarrow)$ Suppose that $x_{(\alpha, \beta, \gamma)}^{e}$ and $y_{\left(\alpha^{\prime}, \beta^{\prime}, \gamma^{\prime}\right)}^{e^{\prime}}$ be two distinct neutrosophic soft points of $(X, \tau, E)$. Then, $x_{(\alpha, \beta, \gamma)}^{e} \subset x_{(1,1,0)}^{e}$ and $y_{\left(\alpha^{\prime}, \beta^{\prime}, \gamma^{\prime}\right)}^{e^{\prime}} \subset y_{(1,1,0)}^{e^{\prime}}$. By hypothesis, $y_{(1,1,0)}^{e^{\prime}}$ and $y_{(1,1,0)}^{e^{\prime}}$ are neutrosophic pre-closed soft sets. Then, $\left[x_{(1,1,0)}^{e}\right]^{c}$ and $\left[y_{(1,1,0)}^{e^{\prime}}\right]^{c}$ are neutrosophic pre-open soft sets such that $x_{(\alpha, \beta, \gamma)}^{e} \in\left[y_{(1,1,0)}^{e^{\prime}}\right]^{c}$, $y_{\left(\alpha^{\prime}, \beta^{\prime}, \gamma^{\prime}\right)}^{e^{\prime}} \in\left[\left[y_{(1,1,0)}^{e^{\prime}}\right]^{c}\right]^{c}$ and $x_{(\alpha, \beta, \gamma)}^{e} \in\left[\left[x_{(1,1,0)}^{e}\right]^{c}\right]^{c}, y_{\left(\alpha^{\prime}, \beta^{\prime}, \gamma^{\prime}\right)}^{e^{\prime}} \in\left[x_{(1,1,0)}^{e}\right]^{c}$. Therefore, $(X, \tau, E)$ is neutrosophic soft pre $T_{1}$.

$(\Leftarrow)$ Suppose that $(X, \tau, E)$ is neutrosophic soft pre $T_{1}$. Let $x_{(1,1,0)}^{e}$ be a neutrosophic soft point with the truth-membership value 1 , the indeterminacy-membership value 1 and falsity- membership value 0 . Take any neutrosophic soft point $y_{\left(\alpha^{\prime}, \beta^{\prime}, \gamma^{\prime}\right)}^{e^{\prime}} \in$ $\left[x_{(1,1,0)}^{e}\right]^{c}$. It is easily seen that $x_{(1,1,0)}^{e}$ and $y_{\left(\alpha^{\prime}, \beta^{\prime}, \gamma^{\prime}\right)}^{e^{\prime}}$ are distinct. From our assumption, there exist neutrosophic pre-open soft sets $(\widetilde{F}, E)$ and $(\widetilde{G}, E)$ such that $x_{(1,1,0)}^{e} \in(\widetilde{F}, E), y_{\left(\alpha^{\prime}, \beta^{\prime}, \gamma^{\prime}\right)}^{e^{\prime}} \in(\widetilde{F}, E)^{c}$ and $x_{(1,1,0)}^{e} \in(\widetilde{G}, E)^{c}, y_{\left(\alpha^{\prime}, \beta^{\prime}, \gamma^{\prime}\right)}^{e^{\prime}} \in(\widetilde{G}, E)$. Then, $y_{\left(\alpha^{\prime}, \beta^{\prime}, \gamma^{\prime}\right)}^{e^{\prime}} \in(\widetilde{G}, E) \subset\left[x_{(1,1,0)}^{e}\right]^{c}$. This means that $\left[x_{(1,1,0)}^{e}\right]^{c}$ is neutrosophic pre-open soft. Therefore, $x_{(1,1,0)}^{e}$ is neutrosophic pre-closed soft. 
Definition 31. A neutrosophic soft topological space $(X, \tau, E)$ is said to be a neutrosophic soft pre $T_{2}$-space if for every pair of distinct neutrosophic soft points $x_{(\alpha, \beta, \gamma)}^{e}, y_{\left(\alpha^{\prime}, \beta^{\prime}, \gamma^{\prime}\right)}^{e^{\prime}}$ there exists neutrosophic pre-open soft sets $(\widetilde{F}, E)$ and $(\widetilde{G}, E)$ such that $x_{(\alpha, \beta, \gamma)}^{e} \in(\widetilde{F}, E)$, $y_{\left(\alpha^{\prime}, \beta^{\prime}, \gamma^{\prime}\right)}^{e^{\prime}} \in(\widetilde{F}, E)^{c}, \quad y_{\left(\alpha^{\prime}, \beta^{\prime}, \gamma^{\prime}\right)}^{e^{\prime}} \in(\widetilde{G}, E), x_{(\alpha, \beta, \gamma)}^{e} \in(\widetilde{G}, E)^{c}$ and $(\widetilde{F}, E) \subset$ $(\widetilde{G}, E)^{c}$.

For a neutrosophic soft topological space $(X, \tau, E)$ we have the following diagram:

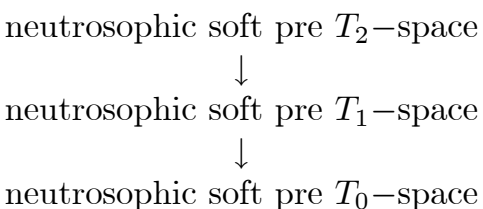

Converse statements may not be true as shown in the examples below;

Example 32. Let $X=\{x, y\}$ be a universe, $E=\{a . b\}$ be a parameteric set and $\left(\widetilde{F}_{a}, E\right)$ be a neutrosophic soft set defined as $\widetilde{F}_{a}(a)=\{\langle x, a, a, 1-a\rangle,\langle y, a, a, 1-a\rangle\}$ and

$\widetilde{F}_{a}(b)=\{\langle x, 0,0,1\rangle,\langle y, a, a, 1-a\rangle\}$ for any $\alpha \in(0,1]$. Then, the family $\tau=\left\{0_{(X, E)}, 1_{(X, E)}\right\} \cup\left\{\left(\widetilde{F}_{a}, E\right): a \in(0,1]\right\}$

is a neutrosophic soft topology over $X$. So, $(X, \tau, E)$ is a neutrosophic soft topological space. $(X, \tau, E)$ is a neutrosophic soft pre $T_{0}$-space but not a neutrosophic soft pre $T_{1}$-space. Because, $x_{(0.9,0.6,0.2)}^{b}$ and $y_{(0.8,0.7,0.4)}^{a}$ are distinct neutrosophic soft points in $(X, \tau, E)$ and there doesn't exist any neutrosophic pre-open soft set that contains $x_{(0.9,0.6,0.2)}^{b}$ but doesn't contain $y_{(0.8,0.7,0.4)}^{a}$.

Example 33. Let $X=\{x, y\}$ be a universe, $E=\{a . b\}$ be a parameteric set and $(\widetilde{F}, E)$ be a neutrosophic soft set defined as $\widetilde{F}(a)=\{\langle x, 0,0,1\rangle,\langle y, 0,0,1\rangle\}$ and $\widetilde{F}(b)=\{\langle x, 0,0,1\rangle,\langle y, 0,0,0.9\rangle\}$. Then, the family $\tau=\left\{0_{(X, E)}, 1_{(X, E)},(\widetilde{F}, E)\right\}$ is a neutrosophic soft topology over $X$. So, $(X, \tau, E)$ is a neutrosophic soft topological space. $(X, \tau, E)$ is a neutrosophic soft pre $T_{1}$-space. But, it is not a neutrosophic soft pre $T_{2}$-space for the existence of distinct neutrosophic soft points $x_{(0.5,0.5,0.1)}^{a}$ and $y_{(0.4,0.4,0.6)}^{b}$.

Theorem 34. Let $(X, \tau, E)$ be a neutrosophic soft topological space. $(X, \tau, E)$ is neutrosophic soft pre $T_{2}$-space if and only if for any pair of distinct neutrosophic 
soft points $x_{(\alpha, \beta, \gamma)}^{e}, y_{\left(\alpha^{\prime}, \beta^{\prime}, \gamma^{\prime}\right)}^{e^{\prime}}$, there exists a neutrosophic pre-open soft set $(\widetilde{F}, E)$ such that

$$
x_{(\alpha, \beta, \gamma)}^{e} \in(\widetilde{F}, E), \quad y_{\left(\alpha^{\prime}, \beta^{\prime}, \gamma^{\prime}\right)}^{e^{\prime}} \in(\widetilde{F}, E)^{c} \text { and } y_{\left(\alpha^{\prime}, \beta^{\prime}, \gamma^{\prime}\right)}^{e^{\prime}} \in[N \operatorname{SPcl}(\widetilde{F}, E)]^{c} \text {. }
$$

Proof. $(\Rightarrow)$ Let $x_{(\alpha, \beta, \gamma)}^{e}$ and $y_{\left(\alpha^{\prime}, \beta^{\prime}, \gamma^{\prime}\right)}^{e^{\prime}}$ be two distinct neutrosophic soft points in $(X, \tau, E)$. Since $(X, \tau, E)$ is a neutrosophic soft pre $T_{2}$-space, there exist two neutrosophic pre-open soft sets $(\widetilde{F}, E)$ and $(\widetilde{G}, E)$ such that $x_{(\alpha, \beta, \gamma)}^{e} \in(\widetilde{F}, E)$, $y_{\left(\alpha^{\prime}, \beta^{\prime}, \gamma^{\prime}\right)}^{e^{\prime}} \in(\widetilde{G}, E)$ and $(\widetilde{F}, E) \subset(\widetilde{G}, E)^{c}$. So, it is implied that $y_{\left(\alpha^{\prime}, \beta^{\prime}, \gamma^{\prime}\right)}^{e^{\prime}} \in$ $(\widetilde{F}, E)^{c}$. Since $(\widetilde{G}, E)^{c}$ is a neutrosophic pre-closed soft set, $\operatorname{NSPcl}(\widetilde{F}, E) \subset$ $(\widetilde{G}, E)^{c}$. This means that, $(\widetilde{G}, E) \subset[N \operatorname{SPcl}(\widetilde{F}, E)]^{c}$. So, $y_{\left(\alpha^{\prime}, \beta^{\prime}, \gamma^{\prime}\right)}^{e^{\prime}} \in[N \operatorname{SPcl}(\widetilde{F}, E)]^{c}$.

$(\Leftarrow)$ Take any pair of distinct neutrosophic soft points $x_{(\alpha, \beta, \gamma)}^{e}, y_{\left(\alpha^{\prime}, \beta^{\prime}, \gamma^{\prime}\right)}^{e^{\prime}}$ in $(X, \tau, E)$. From our assumption, there exists a neutrosophic pre-open soft set $(\widetilde{F}, E)$ such that $x_{(\alpha, \beta, \gamma)}^{e} \in(\widetilde{F}, E), \quad y_{\left(\alpha^{\prime}, \beta^{\prime}, \gamma^{\prime}\right)}^{e^{\prime}} \in(\widetilde{F}, E)^{c}$ and $y_{\left(\alpha^{\prime}, \beta^{\prime}, \gamma^{\prime}\right)}^{e^{\prime}} \in$ $[N S P c l(\widetilde{F}, E)]^{c}$. Since $[N S P c l(\widetilde{F}, E)]^{c}$ is a neutrosophic pre-open soft set and $(\widetilde{F}, E) \subset\left[[N S P c l(\widetilde{F}, E)]^{c}\right]^{c},(X, \tau, E)$ is neutrosophic soft pre $T_{2}$-space.

Theorem 35. A neutrosophic soft subspace $\left(Y, \tau_{Y}, E\right)$ of neutrosophic soft pre $T_{2}-$ space $(X, \tau, E)$ is neutrosophic soft pre $T_{2}$.

Proof. Let $(X, \tau, E)$ be a neutrosophic soft pre $T_{2}$-space, $Y \subseteq X$ and $\left(Y, \tau_{Y}, E\right)$ be a neutrosophic soft subspace. Take any distinct neutrosophic soft points $x_{(\alpha, \beta, \gamma)}^{e}$ and $y_{\left(\alpha^{\prime}, \beta^{\prime}, \gamma^{\prime}\right)}^{e^{\prime}}$ in $\left(Y, \tau_{Y}, E\right)$.

So, these neutrosophic soft points are also contained in $(X, \tau, E)$. Hence, there exist neutrosophic pre-open soft sets $(\widetilde{F}, E)$ and $(\widetilde{G}, E)$ in $\tau$ such that $x_{(\alpha, \beta, \gamma)}^{e} \in$ $(\widetilde{F}, E)$, $y_{\left(\alpha^{\prime}, \beta^{\prime}, \gamma^{\prime}\right)}^{e^{\prime}} \in(\widetilde{G}, E)$ and $(\widetilde{F}, E) \subset(\widetilde{G}, E)^{c}$. Let $(\widetilde{H}, E)$ be a neutrosophic soft set over $Y$ as described in Definition 26. Then, $(\widetilde{H}, E) \cap(\widetilde{F}, E)$ and $(\widetilde{H}, E) \cap(\widetilde{G}, E)$ are neutrosophic pre-open soft sets in $\left(Y, \tau_{Y}, E\right)$ such that $x_{(\alpha, \beta, \gamma)}^{e} \in(\widetilde{H}, E) \cap$ $(\widetilde{F}, E), y_{\left(\alpha^{\prime}, \beta^{\prime}, \gamma^{\prime}\right)}^{e^{\prime}} \in(\widetilde{H}, E) \cap(\widetilde{G}, E)$ and $(\widetilde{H}, E) \cap(\widetilde{F}, E) \subset[(\widetilde{H}, E) \cap(\widetilde{G}, E)]^{c}$. This means that $\left(Y, \tau_{Y}, E\right)$ is neutrosophic soft pre $T_{2}$. 
Definition 36. Let $(X, \tau, E)$ be a neutrosophic soft topological space, $(\widetilde{G}, E)$ be a neutrosophic pre-closed soft set and $x_{(\alpha, \beta, \gamma)}^{e}$ be a neutrosophic soft point such that $x_{(\alpha, \beta, \gamma)}^{e} \in(\widetilde{F}, E)^{c}$. If there exist neutrosophic pre-open soft sets $(\widetilde{G}, E)$ and $(\widetilde{K}, E)$ such that $x_{(\alpha, \beta, \gamma)}^{e} \in(\widetilde{G}, E),(\widetilde{F}, E) \subseteq(\widetilde{K}, E)$ and $(\widetilde{K}, E) \subset(\widetilde{G}, E)^{c}$, then $(X, \tau, E)$ is said to be a neutrosophic soft pre regular space.

Definition 37. A neutrosophic soft topological space $(X, \tau, E)$ is said to be a strong neutrosophic soft pre $T_{1}$-space if every neutrosophic soft point is a neutrosophic preclosed soft set in $(X, \tau, E)$.

Definition 38. A neutrosophic soft pre regular space $(X, \tau, E)$ is said to be a neutrosophic soft pre $T_{3}-$ space if it is also a strong neutrosophic soft pre $T_{1}-$ space.

Theorem 39. Every neutrosophic soft pre $T_{3}$-space is a neutrosophic soft pre $T_{2}-$ space.

Proof. Let $x_{(\alpha, \beta, \gamma)}^{e}$ and $y_{\left(\alpha^{\prime}, \beta^{\prime}, \gamma^{\prime}\right)}^{e^{\prime}}$ be two distinct neutrosophic soft points of a neutrosophic soft pre $T_{3}$-space $(X, \tau, E)$. Then, $y_{\left(\alpha^{\prime}, \beta^{\prime}, \gamma^{\prime}\right)}^{e^{\prime}}$ is neutrosophic preclosed soft set and

$x_{(\alpha, \beta, \gamma)}^{e} \in\left[y_{\left(\alpha^{\prime}, \beta^{\prime}, \gamma^{\prime}\right)}^{e^{\prime}}\right]^{c}$. From the neutrosophic soft pre-regularity, there exist disjoint neutrosophic pre-open soft sets $(\widetilde{G}, E)$ and $(\widetilde{K}, E)$ such that $x_{(\alpha, \beta, \gamma)}^{e} \in$ $(\widetilde{G}, E)$ and

$y_{\left(\alpha^{\prime}, \beta^{\prime}, \gamma^{\prime}\right)}^{e^{\prime}} \subset(\widetilde{K}, E)$. Thus, $x_{(\alpha, \beta, \gamma)}^{e} \in(\widetilde{G}, E)$ and $y_{\left(\alpha^{\prime}, \beta^{\prime}, \gamma^{\prime}\right)}^{e^{\prime}} \in(\widetilde{K}, E)$. Therefore, $(X, \tau, E)$ is neutrosophic soft pre $T_{2}$-space

Theorem 40. A neutrosophic soft subspace $\left(Y, \tau_{Y}, E\right)$ of a neutrosophic soft pre $T_{3}$-space $(X, \tau, E)$ is neutrosophic soft pre $T_{3}$.

Proof. Let $(X, \tau, E)$ be a neutrosophic soft pre $T_{3}$-space, $Y \subseteq X$ and $\left(Y, \tau_{Y}, E\right)$ be a neutrosophic soft subspace. Let $x_{(\alpha, \beta, \gamma)}^{e}$ be any neutrosophic soft point in $\left(Y, \tau_{Y}, E\right)$. It is obvious that $x_{(\alpha, \beta, \gamma)}^{e}$ is also a neutrosophic soft point in $(X, \tau, E)$. Since $(X, \tau, E)$ is a strong neutrosophic soft pre $T_{1}$-space, $x_{(\alpha, \beta, \gamma)}^{e}$ is a neutrosophic pre-closed soft set in $(X, \tau, E)$. Consider the neutrosphic soft set $(\widetilde{H}, E)$ over $Y$ defined in Definition 26. It is easily seen that $(\widetilde{H}, E) \cap x_{(\alpha, \beta, \gamma)}^{e}$ is neutrosophic pre-closed soft in $\left(Y, \tau_{Y}, E\right)$. This means that $\left(Y, \tau_{Y}, E\right)$ is a strong neutrosophic soft pre- $T_{1}$-space. Now, we must show that $\left(Y, \tau_{Y}, E\right)$ is also a neutrosophic soft pre-regular space. Let $(\widetilde{G}, E)$ be a neutrosophic pre-closed soft set in $\left(Y, \tau_{Y}, E\right)$ and $x_{(\alpha, \beta, \gamma)}^{e}$ be a neutrosophic soft point in $\left(Y, \tau_{Y}, E\right)$ such that $x_{(\alpha, \beta, \gamma)}^{e} \in(\widetilde{G}, E)^{c}$. Then, $(\widetilde{G}, E)=(\widetilde{H}, E) \cap(\widetilde{F}, E)$ for some neutrosophic 
pre-closed soft set $(\widetilde{F}, E)$ in $(X, \tau, E)$. Hence, $x_{(\alpha, \beta, \gamma)}^{e} \in[(\widetilde{H}, E) \cap(\widetilde{F}, E)]^{c}$. So, $x_{(\alpha, \beta, \gamma)}^{e} \in(\widetilde{H}, E)^{c} \cup(\widetilde{F}, E)^{c}$. Because of the description of the neutrosophic soft set $(\widetilde{H}, E)$ in Definition 5.2 , it is clear that $x_{(\alpha, \beta, \gamma)}^{e} \notin(\widetilde{H}, E)^{c}$. This means that $x_{(\alpha, \beta, \gamma)}^{e} \in(\widetilde{F}, E)^{c}$. From the neutrosophic soft pre-regularity of $(X, \tau, E)$, there exists neutrosophic pre-open soft sets $(\widetilde{K}, E)$ and $(\widetilde{L}, E)$ such that $x_{(\alpha, \beta, \gamma)}^{e} \in(\widetilde{K}, E),(\widetilde{F}, E) \subseteq(\widetilde{L}, E)$ and $(\widetilde{K}, E) \subseteq(\widetilde{L}, E)^{c}$. This implies that $(\widetilde{H}, E) \cap(\widetilde{K}, E)$ and $(\widetilde{H}, E) \cap(\widetilde{L}, E)$ are neutrosophic pre-open soft sets in $\left(Y, \tau_{Y}, E\right)$ such that $x_{(\alpha, \beta, \gamma)}^{e} \in(\widetilde{H}, E) \cap(\widetilde{K}, E),(\widetilde{F}, E) \subseteq(\widetilde{H}, E) \cap(\widetilde{L}, E)$ and $(\widetilde{H}, E) \cap(\widetilde{K}, E) \subset(\widetilde{H}, E) \cap(\widetilde{L}, E)^{c}$. Therefore, $\left(Y, \tau_{Y}, E\right)$ is neutrosophic soft pre $T_{3}$.

Definition 41. Let $(X, \tau, E)$ be a neutrosophic soft topological space, $\left(\widetilde{F}_{1}, E\right)$ and $\left(\widetilde{F}_{2}, E\right)$ be neutrosophic pre-closed soft sets such that $\left(\widetilde{F}_{1}, E\right) \subset\left(\widetilde{F}_{2}, E\right)^{c}$. If there exist neutrosophic pre-open soft sets $(\widetilde{G}, E)$ and $(\widetilde{K}, E)$ such that $\left(\widetilde{F}_{1}, E\right) \subseteq$ $(\widetilde{G}, E),\left(\widetilde{F}_{2}, E\right) \subseteq(\widetilde{K}, E)$ and $(\widetilde{G}, E) \subset(\widetilde{K}, E)^{c}$, then $(X, \tau, E)$ is said to be a neutrosophic soft pre normal space.

Definition 42. A neutrosophic soft pre normal space $(X, \tau, E)$ is said to be a neutrosophic soft pre $T_{4}$-space, if it is also a strong neutrosophic soft pre $T_{1}-$ space.

Theorem 43. Let $(X, \tau, E)$ be a fuzzy soft topological space. Then, the following statements are equivalent:

(1) $(X, \tau, E)$ is a neutrosophic soft pre normal space.

(2) For every neutrosophic pre closed soft set $(\widetilde{K}, E)$ and neutrosophic preopen soft set $(\widetilde{L}, E)$ such that $(\widetilde{K}, E) \subseteq(\widetilde{L}, E)$, there exists a neutrosophic pre open soft set $(\widetilde{F}, E)$ such that $(\widetilde{K}, E) \subseteq(\widetilde{F}, E), N S P c l(\widetilde{F}, E) \subseteq(\widetilde{L}, E)$.

Proof. (1) $\Rightarrow(2)$ Let $(\widetilde{K}, E)$ be a pre closed soft set and $(\widetilde{L}, E)$ be a fuzzy pre open soft set such that $(\widetilde{K}, E) \subseteq(\widetilde{L}, E)$. Then, $(\widetilde{K}, E),(\widetilde{L}, E)^{c}$ are neutrosophic pre closed soft sets such that $(\widetilde{L}, E)^{c} \subseteq(\widetilde{K}, E)^{c}$. It follows from (1), there exist neutrosophic pre open soft sets $(\widetilde{F}, E)$ and $(\widetilde{G}, E)$ such that $(\widetilde{K}, E) \subseteq(\widetilde{F}, E)$, $(\widetilde{L}, E)^{c} \subseteq(\widetilde{G}, E)$ and $(\widetilde{F}, E) \subseteq(\widetilde{G}, E)^{c}$. Since $(\widetilde{G}, E)^{c}$ is neutrosophic pre 
closed soft, $N \operatorname{SPcl}(\widetilde{F}, E) \subseteq(\widetilde{G}, E)^{c}$. So,

$N S P c l(\widetilde{F}, E) \subseteq(\widetilde{L}, E)$. Therefore, the neutrosophic pre open soft set $(\widetilde{F}, E)$ satisfies the conditions.

$(2) \Rightarrow(1)$ Let $\left(\widetilde{F}_{1}, E\right)$ and $\left(\widetilde{F}_{2}, E\right)$ be neutrosophic pre-closed soft sets such that

$\left(\widetilde{F}_{1}, E\right) \subset\left(\widetilde{F}_{2}, E\right)^{c}$, where $\left(\widetilde{F}_{2}, E\right)^{c}$ is neutrosophic pre open soft. From our hypothesis, there exists a neutrosophic pre open soft set $(\widetilde{F}, E)$ such that $\left(\widetilde{F}_{1}, E\right) \subset$ $(\widetilde{F}, E)$ and

$\operatorname{NSPcl}(\widetilde{F}, E) \subseteq\left(\widetilde{F}_{2}, E\right)^{c}$. So, $\left(\widetilde{F}_{2}, E\right) \subseteq[N \operatorname{SPcl}(\widetilde{F}, E)]^{c},\left(\widetilde{F}_{1}, E\right) \subset(\widetilde{F}, E)$ $[N \operatorname{SPcl}(\widetilde{F}, E)]^{c} \subseteq(\widetilde{F}, E)^{c}$, where $[N \operatorname{SPcl}(\widetilde{F}, E)]^{c}$ and $(\widetilde{F}, E)$ are are neutrosophic pre open soft sets. Thus, $(X, \tau, E)$ is neutrosophic soft pre normal space.

Theorem 44. A neutrosophic pre closed neutrosophic soft subspace $\left(Y, \tau_{Y}, E\right)$ of a neutrosophic soft pre normal space $(X, \tau, E)$ is neutrosophic soft pre normal.

Proof. Let $(\widetilde{F}, E)$ and $(\widetilde{G}, E)$ be neutrosophic pre closed soft sets in $\left(Y, \tau_{Y}, E\right)$ such that $(\widetilde{F}, E) \subset(\widetilde{G}, E)^{c}$. Consider the neutrosphic soft set $(\widetilde{H}, E)$ over $Y$ defined in Definition 26. Then, $(\widetilde{H}, E)$ is neutrosophic pre closed soft in $(X, \tau, E)$, $(\widetilde{F}, E)=(\widetilde{H}, E) \cap(\widetilde{K}, E)$ and $(\widetilde{G}, E)=(\widetilde{H}, E) \cap(\widetilde{L}, E)$ for some neutrosophic pre closed soft sets $(\widetilde{K}, E)$ and $(\widetilde{L}, E)$ in $(X, \tau, E)$. Hence, $(\widetilde{H}, E) \cap(\widetilde{K}, E)$ and $(\widetilde{H}, E) \cap(\widetilde{L}, E)$ are neutrosophic pre closed soft sets in $(X, \tau, E)$ and $(\widetilde{H}, E) \cap$ $(\widetilde{K}, E) \subseteq[(\widetilde{H}, E) \cap(\widetilde{L}, E)]^{c}$. Since $(X, \tau, E)$ is neutrosophic soft pre normal, there exist neutrosophic pre open sets $(\widetilde{M}, E)$ and $(\widetilde{N}, E)$ such that $(\widetilde{H}, E) \cap(\widetilde{K}, E) \subseteq(\widetilde{M}, E),(\widetilde{H}, E) \cap(\widetilde{L}, E) \subseteq(\widetilde{N}, E)$ and $(\widetilde{M}, E) \subseteq(\widetilde{N}, E)^{c}$. So, $(\widetilde{H}, E) \cap(\widetilde{M}, E)$ and $(\widetilde{H}, E) \cap(\widetilde{N}, E)$ are neutrosophic pre open sets in $\left(Y, \tau_{Y}, E\right)$ such that $(\widetilde{F}, E) \subset(\widetilde{H}, E) \cap(\widetilde{M}, E),(\widetilde{G}, E) \subset(\widetilde{H}, E) \cap(\widetilde{N}, E)$ and $(\widetilde{H}, E) \cap(\widetilde{M}, E) \subset[(\widetilde{H}, E) \cap(\widetilde{N}, E)]^{c}$. Therefore, $\left(Y, \tau_{Y}, E\right)$ is neutrosophic soft pre normal.

Definition 45. Let $\left(X, \tau_{1}, E\right),\left(Y, \tau_{2}, K\right)$ be neutrosophic soft topological spaces and 
$f:\left(X, \tau_{1}, E\right) \rightarrow\left(Y, \tau_{2}, K\right)$ be a neutrosophic soft function. The function $f$ is said to be neutrosophic pre irresolute soft, if $f^{-1}((\widetilde{G}, E)) \in \tau_{1}$ for any $(\widetilde{G}, E) \in \tau_{2}$.

Definition 46. Let $\left(X, \tau_{1}, E\right),\left(Y, \tau_{2}, K\right)$ be neutrosophic soft topological spaces and

$f:\left(X, \tau_{1}, E\right) \rightarrow\left(Y, \tau_{2}, K\right)$ be a neutrosophic soft function. The function $f$ is said to be neutrosophic pre irresolute open soft, if $f((\widetilde{F}, E)) \in \tau_{2}$ for any $(\widetilde{F}, E) \in \tau_{1}$.

Theorem 47. Let $\left(X, \tau_{1}, E\right)$ and $\left(Y, \tau_{2}, K\right)$ be neutrosophic soft topological spaces and

$f:\left(X, \tau_{1}, E\right) \rightarrow\left(Y, \tau_{2}, K\right)$ be a neutrosophic soft function which is bijective, neutrosophic pre irresolute soft and neutrosophic pre irresolute open soft. If $\left(X, \tau_{1}, E\right)$ is a neutrosophic soft pre normal space, then $\left(Y, \tau_{2}, K\right)$ is also a neutrosophic soft pre normal space.

Proof. Let $(\widetilde{F}, E)$ and $(\widetilde{G}, E)$ be neutrosophic pre closed soft sets in $\left(Y, \tau_{2}, K\right)$ such that

$(\widetilde{F}, E) \subset(\widetilde{G}, E)^{c}$. Since $f$ is neutrosophic pre irresolute soft, then $f^{-1}((\widetilde{F}, E))$ and

$f^{-1}((\widetilde{G}, E))$ are neutrosophic pre closed soft sets in $\left(X, \tau_{1}, E\right)$ such that

$f^{-1}((\widetilde{F}, E)) \subset\left[f^{-1}((\widetilde{G}, E))\right]^{c}$. Since $\left(X, \tau_{1}, E\right)$ is a neutrosophic soft pre normal space, there exist neutrosophic pre open soft sets $(\widetilde{K}, E)$ and $(\widetilde{L}, E)$ such that

$f^{-1}((\widetilde{F}, E)) \subset(\widetilde{K}, E), f^{-1}((\widetilde{G}, E)) \subset(\widetilde{L}, E)$ and $(\widetilde{K}, E) \subset(\widetilde{L}, E)^{c}$. It follows that

$(\widetilde{F}, E)=f\left[f^{-1}((\widetilde{F}, E))\right] \subset f((\widetilde{K}, E)),(\widetilde{G}, E)=f\left[f^{-1}((\widetilde{G}, E))\right] \subset$ $f((\widetilde{L}, E))$ and $f((\widetilde{K}, E)) \subset f\left((\widetilde{L}, E)^{c}\right)=[f((\widetilde{L}, E))]^{c}$. From the fact that $\mathrm{f}$ is neutrosophic pre irresolute open soft, $f((\widetilde{K}, E))$ and $f((\widetilde{L}, E))$ are neutrosophic pre open soft sets such that $(\widetilde{F}, E) \subset f((\widetilde{K}, E)),(\widetilde{G}, E) \subset f((\widetilde{L}, E))$ and $f((\widetilde{K}, E)) \subset[f((\widetilde{L}, E))]^{c}$. This means that $\left(Y, \tau_{2}, K\right)$ is a neutrosophic soft pre normal space.

\section{Conclusion}

The notions of neutrosophic pre open soft sets, neutrosophic pre closed soft sets, neutrosophic pre soft interior, neutrosophic pre soft closure, neutrosophic soft pre-interior point, neutrosophic soft pre-cluster point and neutrosophic soft pre separation axioms are introduced, and some properties of the notions are studied. Also, several interesting properties have been established. Additionally, a new 
approach is made to the concept of neutrosophic soft topological subspace. Since topological structures on neutrosophic soft sets have been introduced by many scientists, we generalize the pre topological properties to the neutrosophic soft sets which may be useful in some other disciplines. For the existence of compact connections between soft sets and information systems [17,21 the results obtained from the studies on neutrosophic soft topological space can be used to develop these connections. We hope that many researchers will benefit from the findings in this document to further their studies on neutrosophic soft topology to carry out a general framework for their applications in practical life.

\section{REFERENCES}

[1] Al-shami, T.M., Kocinac, Lj.D.R., The equivalence between the enriched and extended soft topologies, Appl. Comput. Math., 18 (2) (2019), 149-162.

[2] Aras, C.G., Sonmez, A., Çakalli, H., An approach to soft functions, J. Math. Anal., 82 (2017), 129-138.

[3] Aras, C.G., Ozturk, T.Y., Bayramov, S., Separation axioms on neutrosophic soft topological spaces, Turk. J. Math., 43 (2019), 498-510.

[4] Atanassov, K., Intuitionistic fuzzy sets, Fuzzy Sets Syst., 20 (1986), 87-96.

[5] Bayramov, S., Gunduz, C., On intuitionistic fuzzy soft topological spaces, TWMS J. Pure Appl. Math., 5 (2014), 66-79.

[6] Bayramov, S., Gunduz, C., A new approach to separability and compactness in soft topological spaces, TWMS J Pure Appl. Math., 9 (2018), 82-93.

[7] Bera, T., Mahapatra, N.K., On neutrosophic soft function, Annals of Fuzzy Mathematics and Informatics, 12 (1) (July 2016), 101-119.

[8] Bera, T., Mahapatra, N.K., Introduction to neutrosophic soft topological space, Opsearch, 54 (2017), 841-867.

[9] Cagman, N., Karatas, S., Enginoglu, S., Soft topology, Comput Math. Appl., 62 (2011), 351-358.

[10] Çakalli, H., Das, P., Fuzzy compactness via summability, Appl. Math. Lett., 22 (11) (2009), $1665-1669$.

[11] Coskun, A.E., Aras, C.G., Cakalli, H., Sonmez, A., Soft matrices on soft multisets in an optimal decision process, AIP Conference Proceedings, 1759, 1, 020099 (2016); doi: $10.1063 / 1.4959713$.

[12] Deli, I., Broumi, S., Neutrosophic soft relations and some Properties, Ann. Fuzzy Math. Inform, 9 (2015), 169-182.

[13] Gunduz, C., Bayramov, S., On the Tietze extension theorem in soft topological spaces, Proceedings of the Institute of Mathematics and Mechanics of the National Academy of Sciences of Azerbaijan, 43 (2017), 105-115.

[14] Hussain, S., On some properties of intuitionistic fuzzy soft boundary, Commun. Fac. Sci. Univ. Ank. Series A1, 69 (2) (2020), 39-50.

[15] Maji, P.K., Neutrosophic soft set, Ann. Fuzzy Math. Inform, 5 (2013), 157-168.

[16] Molodtsov, D., Soft set theory-first results, Comput Math. Appl., 37 (1999), 19-31.

[17] Pei, D., Miao, D., From soft sets to information systems, in: X. Hu, Q. Liu, A. Skowron, T. Y. Lin, R. R. Yager, B. Zhang (Eds.), Proceedings of Granular Computing, in: IEEE 2 (2005), 617-621.

[18] Salma, A.A., Alblowi, S.A., Neutrosophic set and neutrosophic topological spaces, IOSR J. Math., 3 (2012), 31-35.

[19] Shabir, M., Naz, M., On soft topological spaces, Comput Math. Appl., 61 (2011), 1786-1799. 
[20] Smarandache, F., Neutrosophic set, a generalisation of the intuitionistic fuzzy sets, Int. J. Pure Appl. Math., 24 (2005), 287-297.

[21] Xiao, Z., Chen, L., Zhong, B., Ye, S., Recognition for soft information based on the theory of soft sets, in: J. Chen (Ed.), Proceedings of ICSSSM-05, 2 (2005), 1104-1106. 